9. Jiang $\mathrm{HL}$, Xue WJ, Li DQ, Yin AP, Xin X, Li CM, et al. Influence of continuous veno-venous hemofiltration on the course of acute pancreatitis. World J Gastroenterol. 2005;11:4815-21.

10. Zhu Y, Zhang P, Yuan J, He Q, Jiang H, Hu X, et al. Adjunctive continuous high-volume hemofiltration in acute severe pancreatitis patients: a retrospective study. Scand J Gastroenterol. 2009;44:1363-9.

11. Gong D, Zhang P, Ji D, Chen Z, Li W, Li J, et al. Improvement of immune dysfunction in patients withsevere acute pancreatitis by high-volume hemofiltration: a preliminary report. Int J Artif Organs. 2010;33:22-9.

\section{C.M. Romero}

Unidad de Pacientes Críticos, Hospital Clínico Universidad de Chile, Chile

Correo electrónico: caromero@redclinicauchile.cl

\section{Síndrome colinérgico y síndrome intermedio confluyentes en una intoxicación por organofosforados}

\section{Simultaneous cholinergic and intermediate syndromes in organophosphate poisoning}

\section{Sr. Director:}

Se presenta el caso de un varón de 71 años al que su familia traslada a urgencias tras $24 \mathrm{~h}$ de desaparecido. Se observa desorientación en tiempo y espacio e hipertensión, con el resto de las constantes y la exploración física anodinos. La analítica sólo muestra leucocitosis y leve incremento de creatincinasa. El cuadro evoluciona presentando sudoración, hipertensión, trabajo respiratorio con desaturación y descenso del nivel de consciencia. Se orienta inicialmente como edema agudo de pulmón y se realiza TAC craneal para descartar posible accidente cerebrovascular isquémico. Se traslada al paciente a intensivos, donde se lo intuba por progresión del cuadro. En los días siguientes se retira la sedación y la valoración neurológica muestra un Glasgow de 3 , con ausencia de reflejos del tronco y las pupilas mióticas. Mientras se realizan pruebas para determinar otras posibles causas (lesión isquémica del tronco, meningitis, etc.), el paciente despierta y presenta intensa sudoración y salivación que, junto con la miosis y una deposición con intenso olor a hidrocarburos, hacen sospechar intoxicación por organofosforados con síndrome colinérgico. Se solicita la actividad de colinesterasa sérica, que resulta inferior al rango fisiológico y que confirma el diagnóstico. Se inicia el tratamiento con pralidoxima; el paciente evoluciona correctamente, lo que permite la extubación al $5 .^{\circ}$ día de ingreso. En los días siguientes presenta nuevo deterioro clínico muscular con claudicación respiratoria, reintubación e intensificación de la clínica colinérgica, presenta bradicardia extrema refractaria a la reanimación y fallece.

La intoxicación por organofosforados es habitual en zonas agrícolas, pero también forma parte de los constituyentes de productos de uso doméstico. La clínica, producida por inactivación de la enzima colinesterasa, se basa en un conjunto de manifestaciones colinérgicas definidas como muscarínicas, nicotínicas y centrales ${ }^{1,2}$. Desde 1987 Senanavanke y Karalliedde ${ }^{3}$ describen un síndrome que surge de forma brusca después de la fase colinérgica (pasadas $24-96 \mathrm{~h}$ ) en forma de insuficiencia respiratoria grave con parálisis y fasciculaciones de la musculatura proximal de las extremidades y el tórax, y que requiere soporte ventilatorio. Es el denominado síndrome intermedio. El tratamiento de la intoxicación inicial no influye en el curso de este síndrome $e^{2,4}$.

En el caso presentado, además de lo insidioso del cuadro que llevó a diagnósticos erróneos, destaca la confluencia del síndrome colinérgico y el síndrome intermedio, al contrario de lo descrito en la bibliografía médica, donde uno precede al otro.

Además de mantener siempre la sospecha de intoxicación en caso de disminución del nivel de consciencia, en el caso descrito cabe concluir que la desescalada terapéutica requirió una monitorización estricta de las funciones vitales dada la poca predictibilidad en el comportamiento de esta intoxicación.

\section{Bibliografía}

1. Nogué Xarau S, Munné Mas P, Nicolás Arcelis JM. Tratamiento específico de cada intoxicación: organofosforados. En: Morales Torres, editor. s.l. Intoxicaciones agudas. Protocolos de tratamiento. Barcelona: 2003. p. 344-5.

2. Gervilla Caño J, Otal Bareche J, Torres Justribó M, Durán Rabés J. Intoxicación por organofosforados. SEMERGEN. 2007;33:21-3.

3. Senanayake N, Karalliedde L. Neurotoxic effects of organophosphorus insecticides. An intermediate syndrome. N Engl J Med. 1987;316:761-3.

4. De Bleecker J, Vand den Neucker F, Colardyn F. Intermediate syndrome in organophophorus poisoning: A prospective study. Crit Care Med. 1993;21:1706-11.

M. Delgado*, I. Catalán, J. Masclans y A. Mas

Departamento de Medicina Intensiva, Xarxa Assistencial de Manresa, Hospital Sant Joan de Deu de Manresa, Cataluña, España

*Autor para correspondencia.

Correos electrónicos: mdelgmart@yahoo.es (M. Delgado). 